\title{
Two-view Contactless Fingerprint Acquisition Systems: a Case Study for Clay Artworks
}

\author{
Ruggero Donida Labati, IEEE, Member, Angelo Genovese, IEEE, Member \\ Vincenzo Piuri, IEEE, Fellow, Fabio Scotti, IEEE, Member \\ Department of Computer Science \\ Università degli Studi di Milano \\ Milano, 20122, Italy. \\ \{ruggero.donida, angelo.genovese, vincenzo.piuri, fabio.scotti\}@unimi.it
}

\begin{abstract}
The detection of latent fingerprints can be a great aid in determining the authenticity of ancient artworks. In fact, a commonly used technique for the authentication of artworks consists in the comparison of fingerprints present on the surface of an artifact with other available latent fingerprints of an artist. This kind of analysis is particularly important for the authentication of clay artifacts. The clay, in fact, is often modeled barehanded, causing a great number of fingerprints left on the surface. In many cases, the artworks are very valuable or fragile and the latent fingerprints cannot be acquired using classical forensic methods. For this reason, contactless acquisition techniques have been proposed. Most of these techniques are based on the use of a single camera. In single-view acquisition systems, however, it can be difficult to properly estimate the size of the captured area, the obtained images can suffer from problems related to perspective distortions, and a calibration task cannot be performed in all the cases. In this paper, we propose a two-view acquisition system able to capture the latent fingerprints left on a clay artwork, and to compute their threedimensional metric reconstruction. The obtained results show that the proposed approach is feasible and the reconstructed models provide a metric, view-independent, and less-distorted reconstruction of the fingerprint. In particular, we describe the application of the proposed method on a specific clay artwork associated by experts to the famous sculptor Antonio Canova (Italy, 1757-1822).
\end{abstract}

Index Terms-multiple view, three-dimensional reconstruction, latent fingerprint, contactless, clay artwork.

\section{INTRODUCTION}

Clay artworks are often used by the artists to build a preliminary sketch of the sculptures. These artifacts are very important and valuable since they show the artistic path of the authors to the creation of the final artworks.

The authentication of clay artifacts is performed in order to distinguish the original artworks from the numerous forgeries often present in the market in the case of famous sculptors. This process can be made by using different techniques, like the microscope analysis and carbon 14 dating. The authentication is usually performed by art historians in cooperation with forensic scientists. In this context, the analysis of the latent fingerprints present on the artifacts is an important recognition technique. In order to prove the authenticity of a sculpture, the fingerprints present on the artifact can be compared with other latent fingerprints attributed to a specific artist.

However, classical forensic techniques for the acquisition of latent fingerprints, involving the use of films, molds, or dusts, are often impossible to be applied to ancient artworks because of their value and fragility. Another important problem consists in the position of the latent fingerprints, which can be placed on a surface patch that is difficult to reach. For these reasons, contactless acquisition techniques and classical photographic techniques have been used. However, in the case of clay artworks, the perspective distortion present in the captured images can be very high, due to the irregular shape of the object. Moreover, the aging of the artifacts can modify the color of the material, reducing the visibility of the ridge pattern.

In order to limit the presence of perspective deformations in the captured data, multiple view acquisition systems and threedimensional reconstruction techniques can be used to compute a three-dimensional model of the artifact and the fingerprints. The reconstructed models can then be used to perform the authentication by applying algorithms based on two-dimensional or three-dimensional data. In order to compare the fingerprint models with traditional fingerprint images, different unwrapping techniques can be used to obtain the corresponding twodimensional representation of the fingerprint model [1,2]. The ridge pattern should then be enhanced by using techniques for the latent fingerprint analysis [3-5] or systems specifically designed for contactless fingerprint data [1,2,6-8].

In this paper, we propose a low-cost, contactless, twoview acquisition system able to acquire the latent fingerprints left on a clay artwork, and to perform a three-dimensional metric reconstruction of the captured area. In this way, it is possible to obtain a less-distorted reconstruction of the fingerprint with respect to traditional methods. The metric reconstruction permits to determine the size of the model in a view-independent manner. In particular, in this work we focus on the application of the proposed method on a specific clay artwork, attributed by experts to the Italian sculptor Antonio Canova (Italy, 1757-1822), which is probably a sketch of the well-known statue "Ninfa Dormiente" ("Sleeping Nymph") shown at the Victoria and Albert Museum, London.

It can be seen in Fig. 1d-g that the materials constituting the mixture of the clay can create false colors in the ridge structure, which can trick single-view systems or poorlyperformed photographic acquisitions. Moreover, the modeling 
(a)

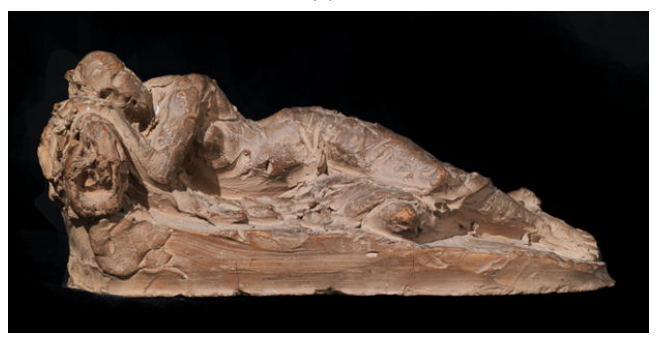

(d)

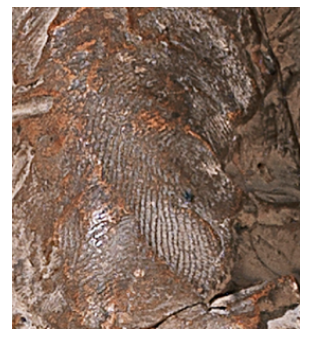

(e)

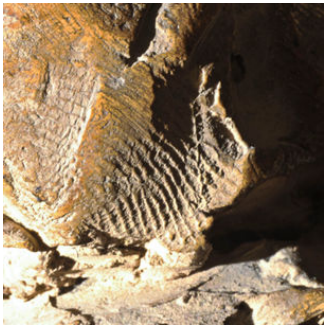

(b)

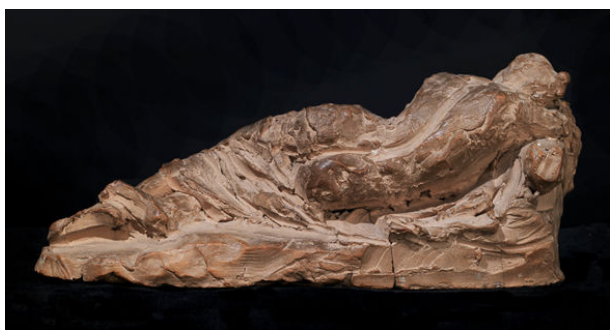

(f)

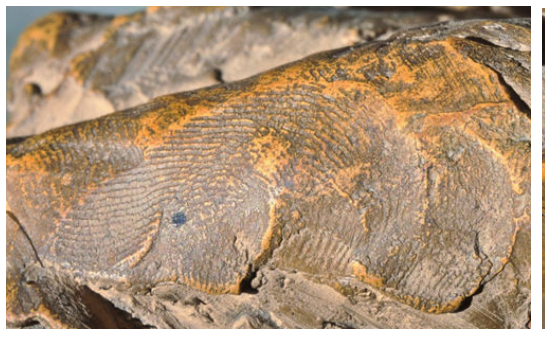

(c)

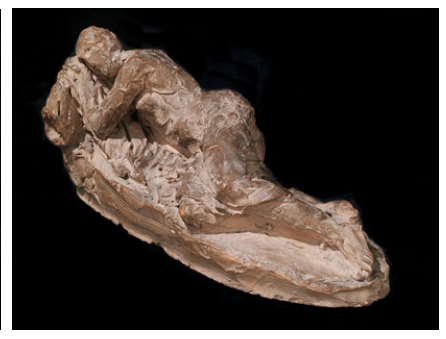

(g)

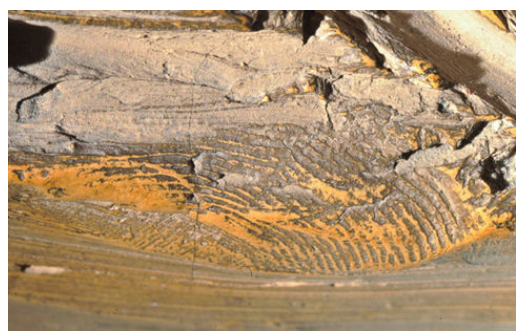

Fig. 1. The studied clay artwork (considered as a working sketch of the "Ninfa Dormiente" statue by Antonio Canova): (a-c) the artwork; (d-g) examples of the artist fingerprints on the surface of the clay acquired with a traditional camera with a macro lense.

process of the artifact produced partial (Fig. 1e) or distorted (Fig. 1f) fingerprints.

\section{PREVIOUS WORK}

One important technique for the authentication of artworks consists in the analysis of latent fingerprints present on the artworks themselves. This analysis is performed by forensic experts and, at the best of our knowledge, there are no automatic methods able to perform a complete automatic authentication. In the literature, there are examples of the use of fingerprints for the authentication of works attributed to important artists, such as Leonardo Da Vinci (Italy, 14521519) $[9,10]$.

In particular, the authentication of statues using latent fingerprints is a complex process because they can present irregular shapes and different levels of degradation. The work described in [11] divides the ancient fingerprints in two-dimensional and three-dimensional impressions. The first class of fingerprints is usually present on hard materials and is due to the presence of chemical substances on the epidermal ridge surfaces. The second class is more present in soft materials, like ceramics or clay, and is related to the imprint of the epidermal ridges into the material. The analysis of three-dimensional impressions using two-dimensional techniques can produce poor results. In both cases, the visibility of the ridge pattern can be reduced by different factors, like the humidity and the aging of the artwork.

Image processing techniques can be used in order to overcome the problems related to the low visibility of the ridge pattern. In the literature, there are many studies on automatic and semi-automatic methods for the enhancement and segmentation of the ridge pattern in latent fingerprint images [3-5].
At the best of our knowledge, however, there are no methods for the three-dimensional reconstruction of latent and ancient fingerprints. Differently, fingerprint recognition systems based on three-dimensional fingertip models of cooperative users are studied. The method described in [1] uses an illumination system shaped as a ring mirror, which captures circular portions of the finger that are subsequently merged. A technique that combines a shape-from-silhouette and a stereoscopic approach is described in [12]: the method uses five cameras placed around the finger and combines the silhouettes of every image in order to approximate the volume of the finger. The images are then merged and superimposed on the volume. The method described in [13] uses a structured light illumination approach in order to compute the three-dimensional reconstruction of the finger. The method described in [2] computes a threedimensional reconstruction of the fingertip using a single twoview acquisition, with the aid of a reference pattern projected onto the finger.

In the literature, there are different three-dimensional reconstruction techniques that can be used for capturing small details of a statue [14]. Contactless techniques should be preferred for precious artworks in order not to damage them. Multiple view techniques [15] are one of the most suitable methods in this applicative context. In fact, other threedimensional reconstruction methods (e.g. techniques based on structured light, or profilometers [16]) require more complex hardware setups, are more difficult to transport, and can be problematic to use for capturing small details of complex surfaces like statues.

\section{THE PROPOSED APPROACH}

A contactless and low-cost two-view acquisition system, and a three-dimensional reconstruction method are proposed in order to capture the areas of the clay artwork containing latent 
(a)

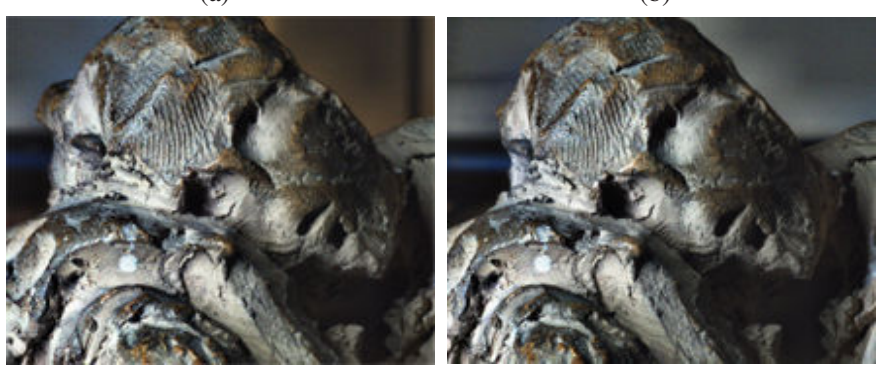

Fig. 2. Example of a two-view acquisition: (a) image A; (b) image B

fingerprints, and to compute the corresponding metric threedimensional models. The resulting model is less-distorted than the single-view acquisitions, and represents a viewindependent, metric reconstruction of the considered region, which allows to determine the size of the latent fingerprint. Moreover, it can be processed using unwrapping algorithms and enhancement techniques.

The proposed method is based on image processing techniques and processes pairs of images captured using two synchronized color CCD cameras. First, a crosscorrelationbased matching technique is used in order to determine a series of matching points in the two images. The points are then triangulated and the three-dimensional model is completed with the wrapping of the interpolated texture computed from the original pair of images.

The proposed method can be divided in the following steps:

1) calibration of the cameras and acquisition of the images;

2) image preprocessing and extraction of the reference points;

3) point matching and triangulation;

4) surface estimation and texture mapping.

\section{A. Calibration of the cameras and acquisition of the images}

The calibration of the cameras is computed off-line, by acquiring multiple views of a chessboard, which are processed using a corner detector algorithm. The intrinsic and extrinsic parameters of the cameras are then computed using the calibration algorithms described in $[17,18]$. The homography matrix is computed using a DLT algorithm described in [15]. A RANSAC algorithm is used to compute the fundamental matrix [19].

The two images are then captured using two synchronized color CCD cameras (Fig. 2).

\section{B. Image preprocessing and extraction of the reference points}

In order to enhance the details of the captured images, an adaptive histogram equalization technique [20] is applied.

Considering the images $I_{A}$ and $I_{B}$ (related to the cameras $\mathrm{A}$ and $\mathrm{B}$, respectively), the three-dimensional reconstruction process requires the search of the points of $I_{B}$ that correspond to a set of points of $I_{A}$. A set of reference points is then selected from the image $I_{A}$ by downsampling the image with a step of $s_{d}$ pixels

\section{Point matching and triangulation}

In the literature, there are approaches specifically designed for the purpose of matching the corresponding pairs of points in a two-view acquisition system, which consider the differences in the orientations of the cameras and illumination conditions [21,22]. Our setup, however, is specifically designed in order to have limited differences in the orientations of the cameras, and the same illumination conditions in the two captured images. For this reason, it is not necessary to apply particularly complex constraints during the matching step. In the literature, there are also approaches designed in order to match the minutiae in contactless fingerprint acquisitions [23]. These approaches, however, cannot be applied in the proposed case study because the considered material presents many irregularities. The proposed matching algorithm is based on the methods described in $[2,24]$ and uses a cross-correlation technique.

As a first step, a preliminary match is computed. For each point $x_{A}$ appertaining to $I_{A}$, the search for the matching point in the second image is performed by using the homography matrix:

$$
X_{B}^{\prime}=H X_{A},
$$

where $H$ represents the $3 \times 3$ homography matrix, $X_{A}$ is the point $x_{A}$ converted in homogeneous coordinates, and $X_{B}^{\prime}$ is the preliminary matching point, expressed in homogeneous coordinates:

$$
X_{B}^{\prime}=\left[\begin{array}{c}
X \\
Y \\
W
\end{array}\right] \text {. }
$$

A Cartesian representation of the point $X_{B}^{\prime}$ is then computed as:

$$
x_{B}^{\prime}=\left[\begin{array}{c}
\frac{X}{W} \\
\frac{Y}{W}
\end{array}\right] .
$$

A series of possible matching points adjacent to $x_{B}^{\prime}$ are extracted in a rectangular region centered in $x_{B}^{\prime}$. The possible matching points are considered if:

$$
\begin{aligned}
& d_{x}\left(x_{B}^{i}, x_{B}^{\prime}\right)<\Delta_{x} \\
& d_{y}\left(x_{B}^{i}, x_{B}^{\prime}\right)<\Delta_{y},
\end{aligned}
$$

where $x_{B}^{i}$ is the $i$-th adjacent point, $d_{x}$ and $d_{y}$ represent the distances in the $x$ and $y$ directions, and $\Delta_{x}$ and $\Delta_{y}$ are the dimensions of the rectangular area.

The distance from the corresponding epipolar line is computed for each of the possible matching points $x_{B}^{i}$ :

$$
d_{e p}^{i}=\frac{\left(X_{B}^{i}\right)^{T} F X_{A}}{\sqrt{\left(l_{1}\right)^{2}+\left(l_{2}\right)^{2}}},
$$

where $d_{e p}^{i}$ is the epipolar distance, $X_{B}^{i}$ is the $i$-th adjacent point expressed in homogeneous coordinates, $F$ is the fundamental matrix, and $l_{1}, l_{2}$ are the first two components of the epipolar line $l$, which is computed using the equation:

$$
l=F X_{A} .
$$


(a)

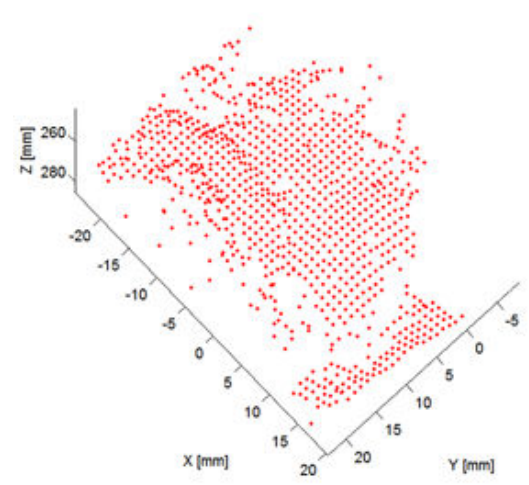

(d)

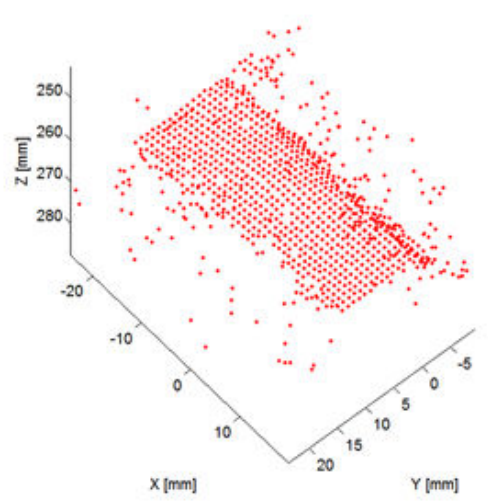

(b)

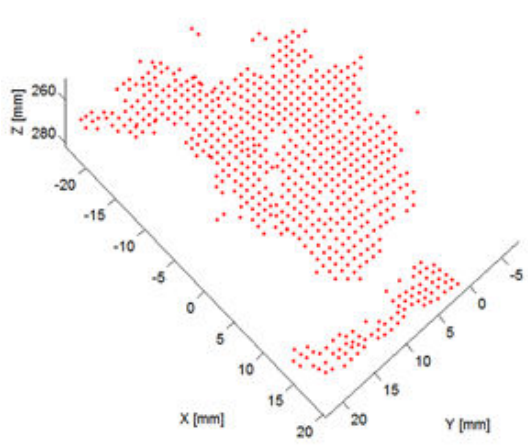

(e)

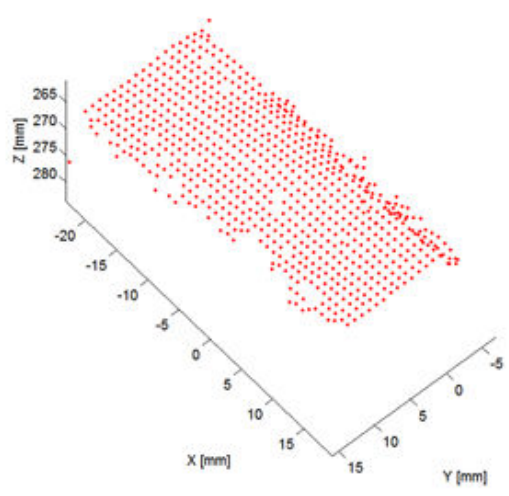

(c)

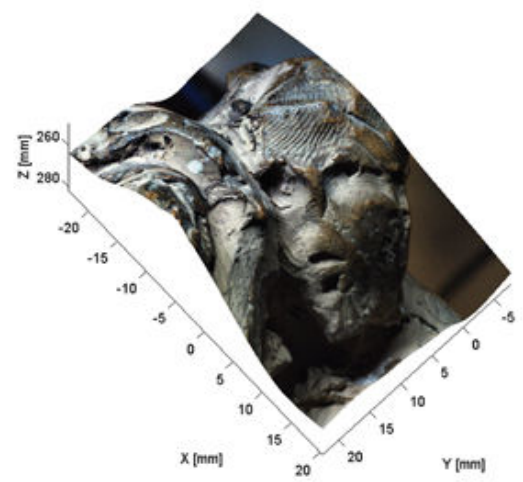

(f)

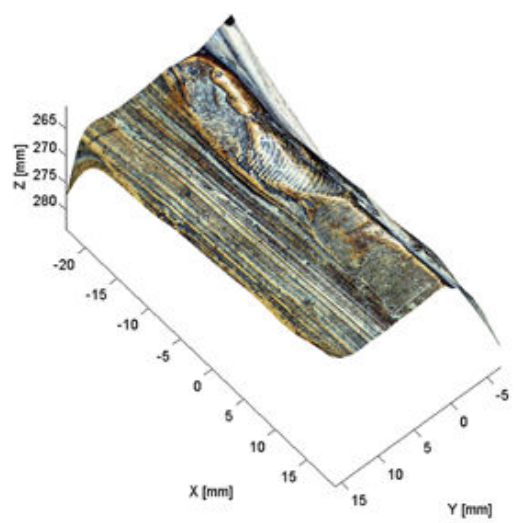

Fig. 3. Examples of reconstructed point clouds of two different acquisitions and the relative texture mappings: (a,d) unfiltered point clouds; (b,e) filtered point clouds; (c,f) mapped textures.

The possible matching points must have an epipolar distance inferior to a threshold $t_{e p}$ :

$$
d_{e p}^{i}<t_{e p}
$$

The Canny edge detector is applied to the two images $I_{A}$ and $I_{B}$ and used as a check for the consistency of the possible matching points. Only the candidate matching points with corresponding values in the binary edge images are considered:

$$
C_{A}\left(x_{A}\right)=C_{B}\left(x_{B}^{i}\right),
$$

where $C_{A}, C_{B}$ are the images resulting from the application of Canny edge detector to $I_{A}$ and $I_{B}$.

The set of possible matching points $x_{B}^{i}$ are inserted in the list $V_{B}$ of the valid points to be checked using the normalized cross-correlation:

$$
x_{B}^{i} \in V_{B} \text { if }\left\{\begin{array}{l}
d_{e p}^{i}<t_{e p}, \\
d_{x}\left(x_{B}^{i}, x_{B}^{\prime}\right)<\Delta_{x}, \\
d_{y}\left(x_{B}^{i}, x_{B}^{\prime}\right)<\Delta_{y}, \\
C_{A}\left(x_{A}\right)=C_{B}\left(x_{B}^{i}\right)
\end{array}\right.
$$

The matching point is then computed by performing the cross-correlation of two $l \times l$ windows, one centered in $x_{A}$, and the others centered in every valid point of $V_{B}$. The crosscorrelation of the two windows is performed on the $Y, R, G, B$ channels separately, and is computed as:

$$
\begin{array}{r}
r=\frac{\sum_{m} \sum_{n}\left(A_{m n}-\bar{A}\right)\left(B_{m n}-\bar{B}\right)}{\sqrt{\left(\sum_{m} \sum_{n}\left(A_{m n}-\bar{A}\right)^{2}\right)\left(\sum_{m} \sum_{n}\left(B_{m n}-\bar{B}\right)^{2}\right)}}, \\
1<m<l, \quad 1<n<l,
\end{array}
$$

where $A$ and $B$ are the two windows of size $l \times l$. The final matching point $x_{B}$ is chosen as the one which produces the highest cross-correlation coefficient.

The pairs of matched points are then rectified using the calibration data. Then, the $z$ coordinates of each pair of points are computed using a triangulation equation:

$$
z=\frac{f T}{d\left(x_{A}, x_{B}\right)},
$$

where $f$ is the focal length of the two cameras, $T$ is the translation vector describing the baseline distance between the two cameras, $x_{A}$ and $x_{B}$ are the two matched points, and $d$ is the function that represents the Euclidean distance.

Since the points are obtained using a downsample method with a constant step $s_{d}$, and the surface is sufficiently smooth, 
the three-dimensional point cloud of the reconstructed model should than present a regular distribution of the points. A check for outliers is then performed by removing the threedimensional points which are not close to any other point of the point cloud. The distance from each point and the points appertaining to its 4-neighborhood must be inferior to a threshold $t_{d}$ :

$$
d\left(\left(x_{i}, y_{i}, z_{i}\right),\left(x_{i+j}, y_{i+j}, z_{i+j}\right)\right)<t_{d} ; 1 \leq j \leq 4,
$$

where $\left(x_{i}, y_{i}, z_{i}\right)$ is the $i$-th three-dimensional point, $\left(x_{i+j}, y_{i+j}, z_{i+j}\right)$ are the points appertaining to its 4neighborhood, and $d$ represents the Euclidean distance.

The threshold $t_{d}$ is computed as the double of the minimum distance between adjacent three-dimensional points:

$$
t_{d}=2 \min _{i=1 \ldots N}\left(d\left(\left(x_{i}, y_{i}, z_{i}\right),\left(x_{i+1}, y_{i+1}, z_{i+1}\right)\right)\right),
$$

where $N$ is the number of three-dimensional points.

\section{Surface estimation and texture mapping}

The reconstructed model is merged in a single point cloud $(X, Y, Z)$ and the intensity values of the original image $I_{A}$ are stored in the vector $C$. From the vectors $X, Y$, the maps $S_{x}$ and $S_{y}$ are computed as a mesh with a constant step $s_{\text {interp }}$. The surface map $S_{z}$ and the intensity map $S_{c}$ are then obtained applying a bilinear interpolation on the vectors $Z$ and $C$ at the coordinates described by the meshed maps $S_{x}$ and $S_{y}$. Examples of reconstructed point clouds with the relative estimated surfaces and wrapped textures are shown in Fig. 3.

\section{EXPERIMENTAL RESUlts}

We used two Sony XCD-SX90CR CCD color cameras synchronized using a trigger mechanism for capturing the images of the artwork. The angle of the cameras with respect of the horizontal support is $\alpha=85^{\circ}$, and the baseline distance between the cameras is $\Delta_{D}=45 \mathrm{~mm}$ (considering the centers of the CCDs). The distance from the cameras to the surface of the statue is about $\Delta_{H}=205 \mathrm{~mm}$, chosen according to the focal planes of the two cameras. The intersection of the focal planes of the two cameras was guaranteed by mounting on the cameras two lasers that projected two vertical lines during the mounting step of the setup. The intersection of the vertical lines was set in order to correspond to the region of the acquisition volume with the best optical focus. Fig. 4 shows the schema of the used acquisition setup.

We used 15 pairs of chessboard acquisitions to calibrate the cameras. The chessboard is composed by $12 \times 9$ squares of $2.8 \times 2.8 \mathrm{~mm}$. We used these images also to estimate a calibration error, computed by reconstructing the point clouds of the chessboard and interpolating a plane through the threedimensional points. The error measure is defined as the standard deviation of the distances of the reconstructed points from the interpolating plane [25], and is equal to $0.04 \mathrm{~mm}$. In order to measure the accuracy of the proposed method, we captured also a semi-sphere of $20 \mathrm{~mm}$ radius and reconstructed the corresponding three-dimensional models. Then, we computed

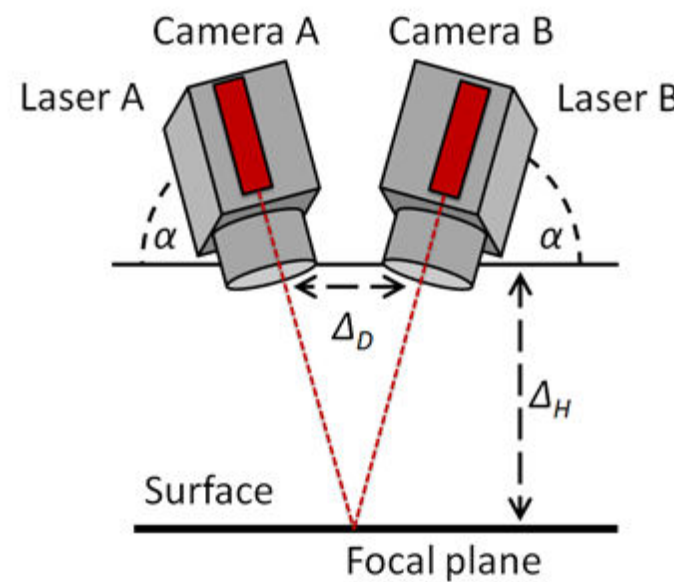

Fig. 4. Schema of the proposed acquisition setup.

the difference between the radius of the sphere fitted using the three-dimensional reconstructed points and the actual radius. The resulting reconstruction error is equal to $0.5 \mathrm{~mm}$.

We captured a total of 275 pairs of images with different points of view and illumination conditions. We used a led illumination only for enhancing the visibility of the particular area of the surface. Otherwise, no specific illumination system was designed. These images represent all the ancient fingerprints present on the surface of the clay artwork.

The majority of the captured images describe impressions that cannot be used to perform an identification since they represent very small regions of the finger or are affected by problems related to deformations and aging of the surface of the statue. In some acquisitions, the reconstructed samples can be probably sufficient to try an authentication procedure with different clay artworks of the same author, even if the matching can be done only on fragments and not by using complete fingerprints.

We applied the proposed method on the captured twoview images and reconstructed the corresponding metric threedimensional models. Fig. 5, shows some examples of the reconstructed models of the regions containing latent fingerprints. It is possible to observe that three-dimensional models of a fingerprint area permit to obtain a view-independent, metric reconstruction of the fingerprint, and to compensate to the problems related to distortions and different camera orientations.

The fingerprint can then be analyzed using a more suitable point of view. Fig. 6 shows an example of captured pairs of images and the corresponding three-dimensional models. It is possible to observe how the three-dimensional models help in creating less-distorted acquisitions of the fingerprints. In order to compute the same viewpoint, the models were registered using an Iterative Closest Point algorithm [26], which computes the rotation and translation of a model, with respect to another point cloud. The computed transformations were applied on one of the reconstructed point clouds, using 
(a)

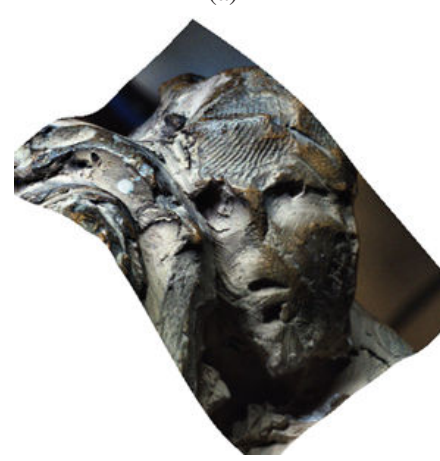

(e)

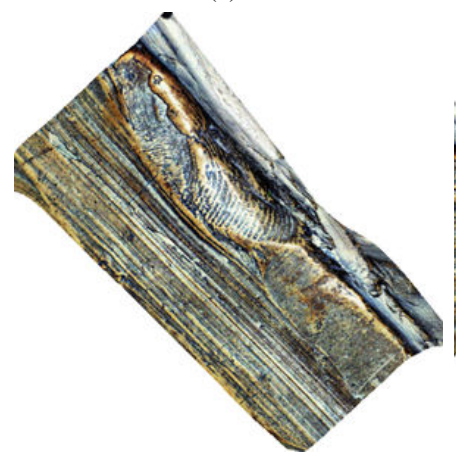

(i)

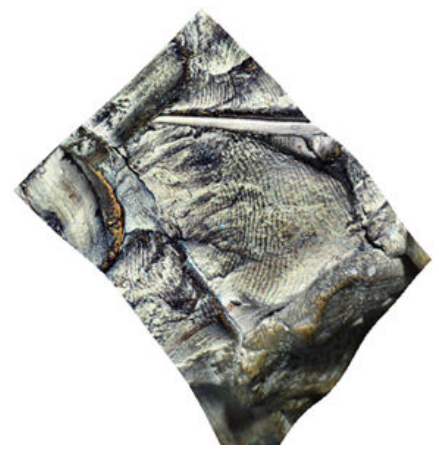

(b)

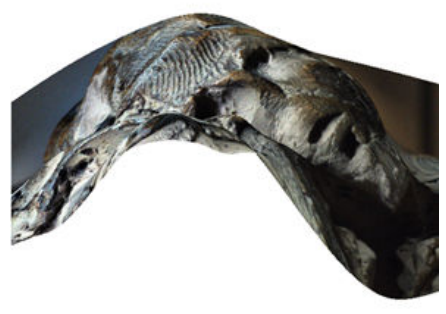

(f)

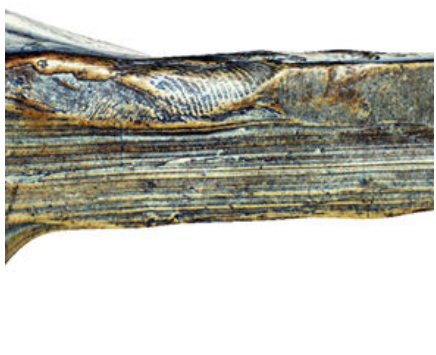

(j)

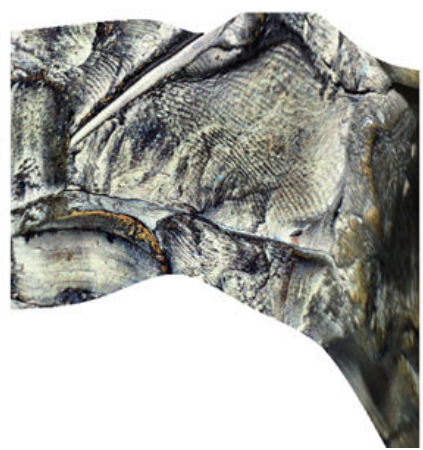

(c)

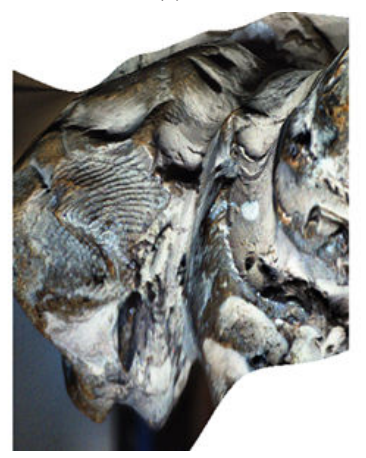

(g)

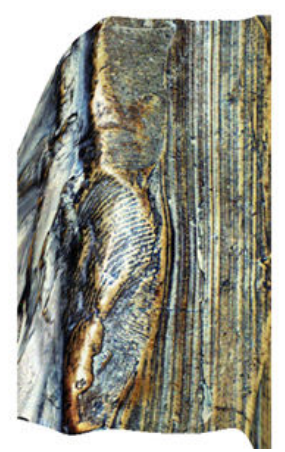

(k)

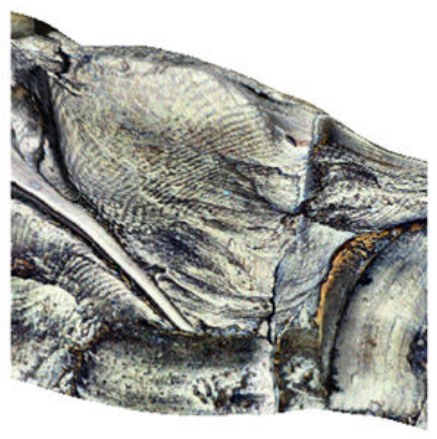

(d)

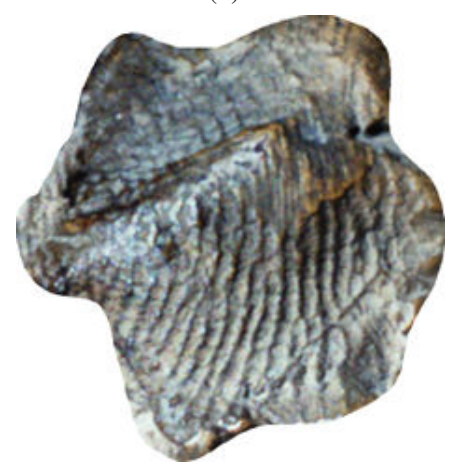

(h)

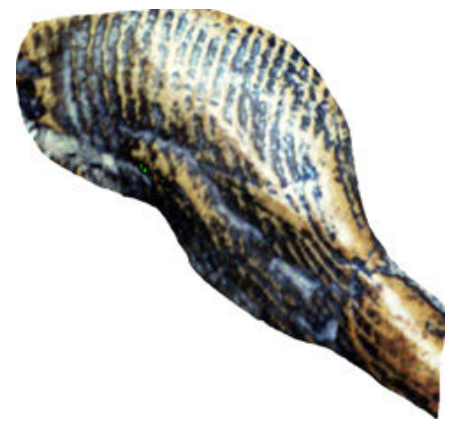

(1)

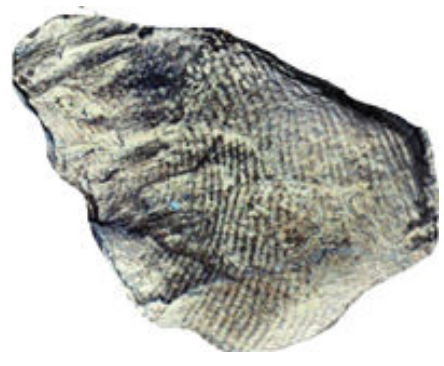

Fig. 5. Examples of reconstructed three-dimensional models plotted with different orientations: (a,e,i) models seen from the first point of view; (b,f,j) models seen from a second point of view; $(\mathrm{c}, \mathrm{g}, \mathrm{l})$ models seen from a third point of view; $(\mathrm{d}, \mathrm{h})$ particulars of the reconstructed latent fingerprint.

the equation:

$$
P_{i}^{\prime}=R P_{i}+T
$$

where $P_{i}=\left(x_{i}, y_{i}, z_{i}\right), R$ is the $3 \times 3$ rotation matrix, and $T$ is the $3 \times 1$ translation vector. It is possible to observe that the three-dimensional models obtained by the proposed method are effectively less distorted and independent by the point of view of the acquisition.

Another example of registered three-dimensional models obtained using the proposed method is shown in Fig. 7. Despite Fig. 7 depicts three-dimensional models representing only a partial fingerprint, Fig. 7e and Fig. 7f are a clear example of the capability of the proposed method to obtain aligned, view-independent portions of clay atifacts.

Metric fingerprint samples independent by the point of view can also be obtained by using other acquisition techniques based on single images. These techniques, however, require complex hardware setups and are difficult to apply in the evaluated context.

The three-dimensional models obtained by the proposed method can then be used in order to perform biometric recognitions. For example, it is possible to apply matching techniques based on traditional two-dimensional images or directly on three-dimensional data [1]. Matching techniques based on two-dimensional templates can guarantee the compatibility of the fingerprint models with fingerprint images captured using touch-based sensors or traditional forensic techniques. However, they require the estimation of an equivalent twodimensional representation of the fingerprint from the threedimensional model. The computation of this fingerprint representation should be performed by considering the shape of the surface in which the latent fingerprint is present. Considering 
(a)

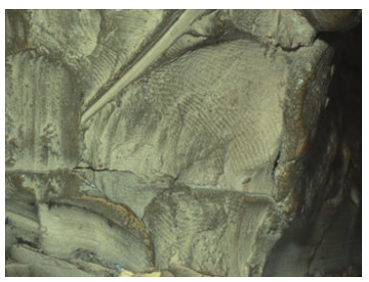

(e)

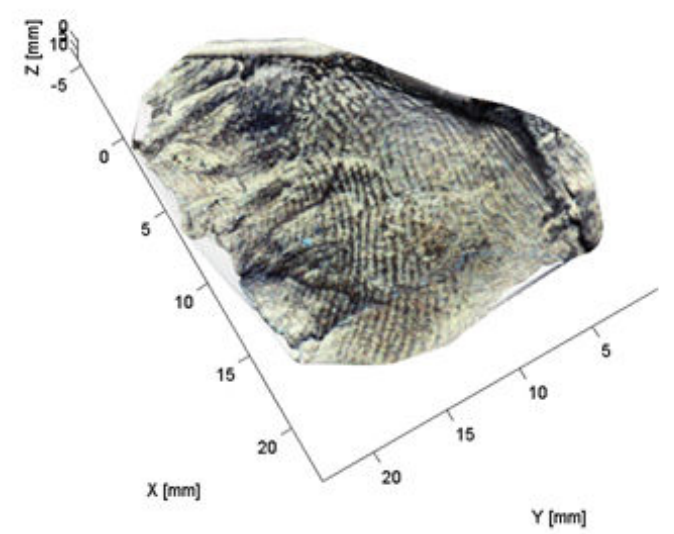

(c)

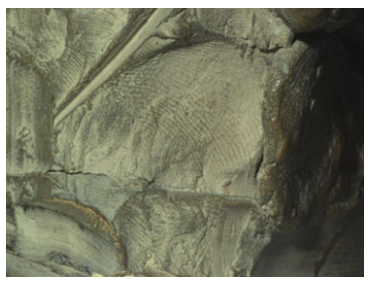

)
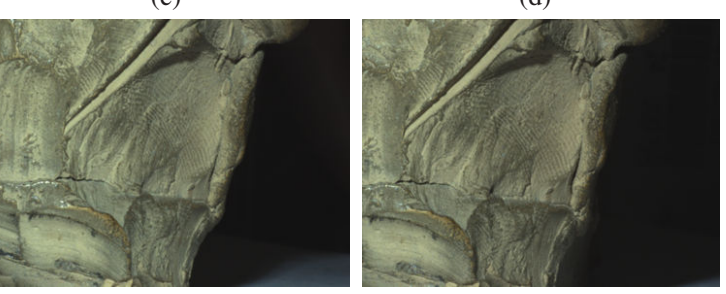

(f)

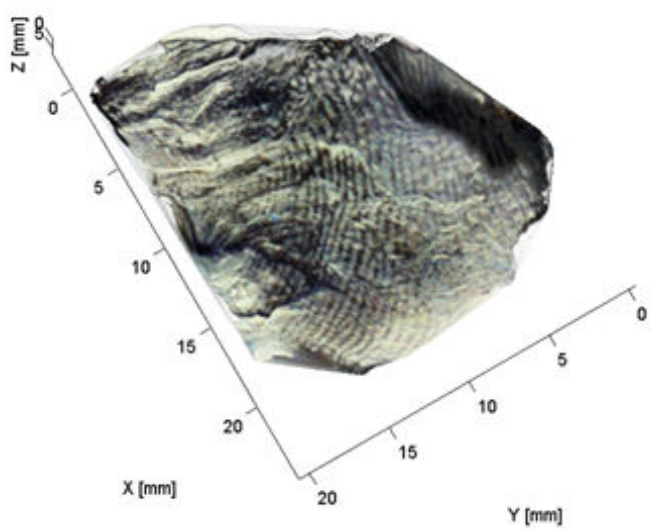

Fig. 6. Examples of captured pairs of images and corresponding three-dimensional models: pair of images 1 (a, b), pair of images 2 (c, d), three-dimensional model 1 (e), three-dimensional model 2 (f). It is possible to observe that the use of three-dimensional models reduces perspective problems related to different points of view and provides a robust metric reconstruction of the fingerprint.

(a)

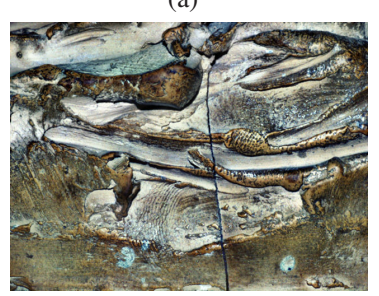

(e)

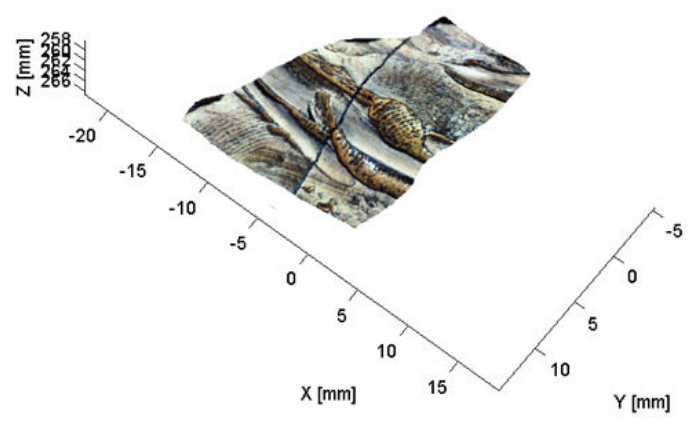

(c)

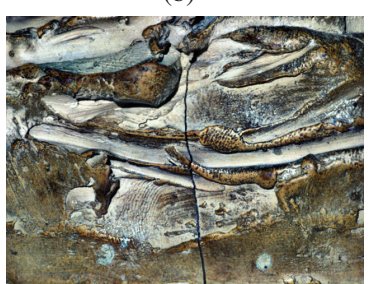

)

Y

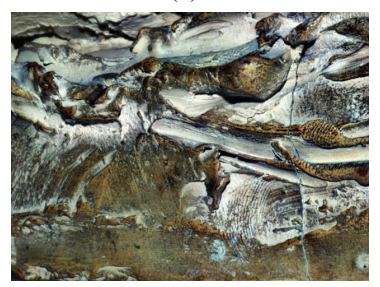

(f)

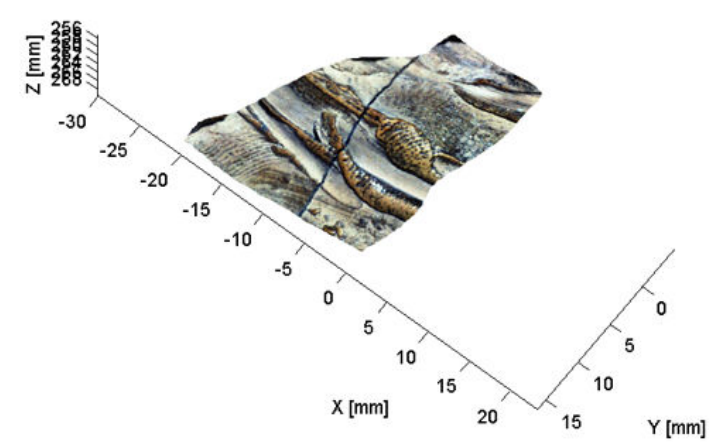

Fig. 7. Examples of captured pairs of images and corresponding three-dimensional models: pair of images 1 (a, b), pair of images 2 (c, d), three-dimensional model 1 (e), three-dimensional model 2 (f). It is possible to observe that the three-dimensional models are independent from the point of view. 
flat surfaces with two-dimensional impressions, in fact, it can be sufficient to extract the texture of the three-dimensional model. More complex surfaces require the use of unwrapping techniques. The technique presented in [2], for example, is designed for cylindrical shaped objects.

\section{Conclusions}

In this paper, we proposed a contactless and low-cost twoview acquisition method, and a three-dimensional reconstruction technique able to capture a pair of images of a latent fingerprint left on a clay artwork and to estimate a threedimensional metric model of the captured area. The proposed method permits to obtain a less-distorted representation of the fingerprint, with respect to single-view acquisitions achieved by classical photographic techniques. The computed models have the advantages of allowing a view-independent and metric reconstruction, which is difficult to obtain using singleview acquisition setups.

The method is based on the extraction of a series of reference points from an image. The corresponding points in the second image are then matched using an algorithm based on the normalized cross-correlation. The three-dimensional coordinates of the matched pairs of points are then computed and an outlier removal algorithm is applied. The resulting model is completed by applying a surface interpolation technique and performing a texture mapping procedure.

The accuracy of the proposed method was evaluated using objects with known shapes. Then, the quality of the threedimensional models computed using the proposed method was inspected by a visual analysis and then compared with the images captured using a single-view system. The obtained results showed that the proposed approach is able to compute a less-distorted, metric and view-independent representation of the latent fingerprints left on the artwork, using a contactless low-cost acquisition setup.

\section{ACKNOWLEDGEMENTS}

The authors wish to thank Carlo Virgilio and Stefano Grandesso from "Arte Moderna e Contemporanea”, Rome, Italy, for the possibility to produce the acquisitions of the artwork, as well for the profitable discussions about the artistic and historical context needed to face effectively this specific application of the proposed method.

\section{REFERENCES}

[1] G. Parziale and Y. Chen, "Advanced technologies for touchless fingerprint recognition," in Handbook of Remote Biometrics, ser. Advances in Pattern Recognition, M. Tistarelli, S. Z. Li, and R. Chellappa, Eds. Springer London, 2009, pp. 83-109.

[2] R. Donida Labati, A. Genovese, V. Piuri, and F. Scotti, "Fast 3-D fingertip reconstruction using a single two-view structured light acquisition," in 2011 IEEE Workshop on Biometric Measurements and Systems for Security and Medical Applications (BIOMS), September 2011, pp. 1-8.

[3] S. Yoon, J. Feng, and A. K. Jain, "On latent fingerprint enhancement," Biometric Technology for Human Identification VII, vol. 7667, no. 1, 2010.

[4] _ - "Latent fingerprint enhancement via robust orientation field estimation," International Joint Conference on Biometrics (IJCB), pp. 1-8, 2011.
[5] F. Chen, J. Feng, A. Jain, J. Zhou, and J. Zhang, "Separating overlapped fingerprints," IEEE Transactions on Information Forensics and Security, vol. 6, no. 2, pp. 346-359, June 2011.

[6] R. Donida Labati, A. Genovese, V. Piuri, and F. Scotti, "Quality measurement of unwrapped three-dimensional fingerprints: a neural networks approach," in 2012 International Joint Conference on Neural Networks (IJCNN), June 2012.

[7] R. Donida Labati, V. Piuri, and F. Scotti, "Measurement of the principal singular point in fingerprint images: a neural approach," in 2010 IEEE International Conference on Computational Intelligence for Measurement Systems and Applications (CIMSA), September 2010, pp. 18-23, 978-1-4244-7228-4.

[8] _ , "Neural-based quality measurement of fingerprint images in contactless biometric systems," in The 2010 International Joint Conference on Neural Networks (IJCNN), July 2010, pp. 1-8.

[9] J. Daab, "Forensic science and fine art authentication, 'la bella principessa'," Fine art registry. [Online]. Available: http://www.fineartregistry.com/articles/ fine-art-forensics/la-bella-principessa-case-study.php

[10] D. Grann, "The mark of a masterpiece," The New Yorker, 2010. [Online]. Available: http://www.newyorker.com/reporting/2010/07/12/ 100712fa_fact_grann

[11] M. Králík, "Fingerprints on artifacts and historical items: examples and comments," Journal of Ancient Fingerprints, 2007.

[12] G. Parziale, E. Diaz-Santana, and R. Hauke, "The surround imager: A multi-camera touchless device to acquire $3 \mathrm{D}$ rolled-equivalent fingerprints." in ICB'06, 2006, pp. 244-250.

[13] Y. Wang, L. Hassebrook, and D. Lau, "Data acquisition and processing of 3-D fingerprints," IEEE Transactions on Information Forensics and Security, vol. 5, no. 4, pp. 750-760, December 2010.

[14] C. Nitschke, 3D Reconstruction - Real-time Volumetric Scene Reconstruction from Multiple Views. VDM Verlag Dr. Muller, April 2007.

[15] R. I. Hartley and A. Zisserman, Multiple View Geometry in Computer Vision, 2nd ed. Cambridge University Press, ISBN: 0521540518, 2004.

[16] B. Curless, "From range scans to 3D models," SIGGRAPH Computer Graphics, vol. 33, no. 4, pp. 38-41, November 1999.

[17] Z. Zhang, "A flexible new technique for camera calibration," IEEE Transactions on Pattern Analysis and Machine Intelligence, vol. 22, pp. 1330-1334, November 2000.

[18] J. Heikkila and O. Silven, "A four-step camera calibration procedure with implicit image correction," in Proceedings of the 1997 Conference on Computer Vision and Pattern Recognition, ser. CVPR. Washington, DC, USA: IEEE Computer Society, 1997, pp. 1106-1112.

[19] P. D. Kovesi, "MATLAB and Octave functions for computer vision and image processing," Centre for Exploration Targeting, School of Earth and Environment, The University of Western Australia, available from: $<$ http://www.csse.uwa.edu.au/ pk/research/matlabfns/ $>$.

[20] R. C. Gonzalez and R. E. Woods, Digital Image Processing (3rd Edition). $\quad$ Upper Saddle River, NJ, USA: Prentice-Hall, Inc., 2006.

[21] Y. S. Heo, K. M. Lee, and S. U. Lee, "Robust stereo matching using adaptive normalized cross-correlation," IEEE Transactions on Pattern Analysis and Machine Intelligence, vol. 33, no. 4, pp. 807-822, April 2011.

[22] J. Sarvaiya, S. Patnaik, and S. Bombaywala, "Image registration by template matching using normalized cross-correlation," in ACT '09, December 2009, pp. 819-822.

[23] R. Donida Labati, V. Piuri, and F. Scotti, "A neural-based minutiae pair identification method for touchless fingeprint images," in IEEE Symposium Series in Computational Intelligence (SSCI), April 2011.

[24] R. Donida Labati, A. Genovese, V. Piuri, and F. Scotti, "Low-cost volume estimation by two-view acquisitions: A computational intelligence approach," in 2012 International Joint Conference on Neural Networks (IJCNN), June 2012.

[25] R. Guerchouche and F. Coldefy, "Camera calibration methods evaluation procedure for images rectification and 3D reconstruction," in Proceedings of the 16th International Conference in Central Europe on Computer Graphics, Visualization and Computer Vision (WSCG'2008), February 2008, pp. 205-210.

[26] P. Besl and H. McKay, "A method for registration of 3-D shapes," IEEE Transactions on Pattern Analysis and Machine Intelligence, vol. 14, no. 2, pp. 239-256, February 1992. 